\title{
Failure of Cardiac Pacemaker to Capture
}

National Cancer Institute

\section{Source}

National Cancer Institute. Failure of Cardiac Pacemaker to Capture. NCI Thesaurus. Code C99527.

Failure to capture manifests as a high pacing threshold that results in either intermittent failure to capture at maximal programmed output or excessive battery drain leading to premature battery exhaustion. 